\title{
Dependence of Tunneling Ionization and Harmonic Generation on the Structure of Molecules by Short Intense Laser Pulses
}

\author{
C. D. Lin \\ J. R. Macdonald Laboratory, Physics Department, Kansas State University, \\ Manhattan, Kansas 66506-2604, USA \\ X. M. Tong \\ Institute of Materials Science, Graduate School of Pure and Applied Science, \\ University of Tsukuba, 1-1-1 Tennodai, Tsukuba, Ibaraki 305-8573, Japan, and, \\ Center for Computational Sciences, University of Tsukuba, 1-1-1 Tennodai, \\ Tsukuba, Ibaraki 305-8577, Japan
}

\begin{abstract}
We illustrate how the sub-10fs lasers and attosecond XUV pulses can be used to probe the structure of molecules. Experimental results of the alignment dependence of the ionization rates and the high-order harmonic generation yields are shown to reflect the structure of the highest occupied molecular orbital. We also show that the vibrational wave packet of simple molecules can be mapped accurately, including the broadening and interference, by using attosecond light pulses.
\end{abstract}

Key words: Tunneling Ionization rates; harmonic generation; attosecond pulses; vibrational wave packet; molecular alignment.

\section{Introduction}

When neutral molecules are exposed to intense infrared laser pulses of sufficiently high intensity, they undergo single or multiple ionization followed by immediate dissociation. Many earlier experiments have shown that the ionized products are strongly forward peaked in the direction of the laser polarization.

Email address: cdlin@phys.ksu.edu (C. D. Lin). 
The nature and the mechanism for resulting in such strong anisotropic angular distributions of the final ion fragments, however, continues to be a subject of debate since the 1990's. Specifically it is known for long time that neutral molecules can be dynamically or adiabatically aligned before ionization by the laser [1]. Furthermore, the ionization rates of molecules by lasers are expected to depend on the orientation and alignment of molecules. Continuing development of laser technology in the last decade has pushed laser pulses of durations to tens of femtoseconds, and more recently, to as short as 5 fs or 4 fs. In the same time, multi-particle detectors such as COLTRIMS have been widely used to measure simultaneously the full 3D momenta of all the ion products. These new measurements have enabled experimentalists to map out the detailed dynamics of simple molecules in the laser field, and interestingly, a number of basic structural features of simple molecules have thus been revealed. In this paper we review several of these recent accomplishments and show how these results have been quantitatively explained by theoretical calculations.

\section{Probing molecular orbital by measuring the alignment depen- dence of ionization rates}

For Ti-Sapphire lasers with peak intensity of the order of $10^{14} \mathrm{~W} / \mathrm{cm}^{2}$, tunneling ionization is the main mechanism for the ionization of atoms and molecules. Since the electronic charge density in a molecule is not isotropic it is clear that the ionization rates of molecules in an intense laser field will depend on the alignment of molecules with respect to the laser polarization direction. For many years such alignment dependence cannot be readily evaluated until the molecular tunneling ionization theory (MO-ADK)[2] was developed recently. The tunneling ionization theory for atoms has been in existence for many years. In the version for ionization by laser fields, in the so-called ADK (Ammosov-Delone-Krainov) model [3] the ionization rate is given analytically. The MO-ADK theory for the ionization of molecules is also given analytically. It further predicts simple alignment dependence of the ionization rates.

For an atom, the wavefunction of the valence electron at large distance where tunneling occurs can be written as

$$
\Psi^{m}(\mathbf{r})=C_{l} F_{l}(r) Y_{l m}(\hat{\mathbf{r}})
$$

where

$$
F_{l}(r \rightarrow \infty) \approx r^{Z_{c} / \kappa-1} e^{-\kappa r}
$$

with $Z_{c}$ the effective Coulomb charge, $\kappa=\sqrt{2 * I_{p}}$, and $I_{p}$ is the ionization en- 
ergy. To employ the ADK formula directly for molecules, one needs to expand the wavefunction in the asymptotic region in terms of one-center expressions. Thus one writes the molecular wavefunction in the tunneling region as

$$
\Psi^{m}(\mathbf{r})=\sum_{l} C_{l} F_{l}(r) Y_{l m}(\hat{\mathbf{r}})
$$

where the summation over $\ell$ is needed. The coefficients $C_{l}$ are obtained by fitting the asymptotic molecular wavefunction. They depend on the internuclear separation and on the electronic state. Once the coefficients $C_{l}$ are available the ionization rate for a diatomic molecule with its axis aligned along the laser polarization is given by

$$
w_{\text {stat }}(F, 0)=\frac{B^{2}(m)}{2^{|m|}|m| !} \frac{1}{\kappa^{2 Z_{c} / \kappa-1}}\left(\frac{2 \kappa^{3}}{F}\right)^{2 Z_{c} / \kappa-|m|-1} e^{-2 \kappa^{3} / 3 F},
$$

where

$$
B(m)=\sum_{l} C_{l} Q(l, m)
$$

and

$$
Q(l, m)=(-1)^{m} \sqrt{\frac{(2 l+1)(l+|m|) !}{2(l-|m|) !}} .
$$

Clearly the factor $\mathrm{B}^{2}(\mathrm{~m})$ measures the electron density in the tunneling region along the direction of the electric field. If the molecular axis is not aligned along the field direction, then eq. (3) should be transformed using the rotational function.

An immediate consequence of the MO-ADK theory is that the ionization rates for any space-fixed molecules are proportional to the electron density in the direction of the laser polarization direction. In other words, by measuring the alignment dependence of the ionization rates, the electron density of the outermost molecular orbital can be directly probed. This is the most significant prediction of the MO-ADK theory.

Since molecules are not easily aligned, the most straightforward way to measure the direction of the molecule at the time of ionization is when the ionized molecules break up into two fragmented ions, where the events are clearly identified when their momenta are summed to zero. Such experiments are routinely employed in experiments using COLTRIMS apparatus where the momentum of each individual fragmented ion is measured over $4 \pi$ angles. 

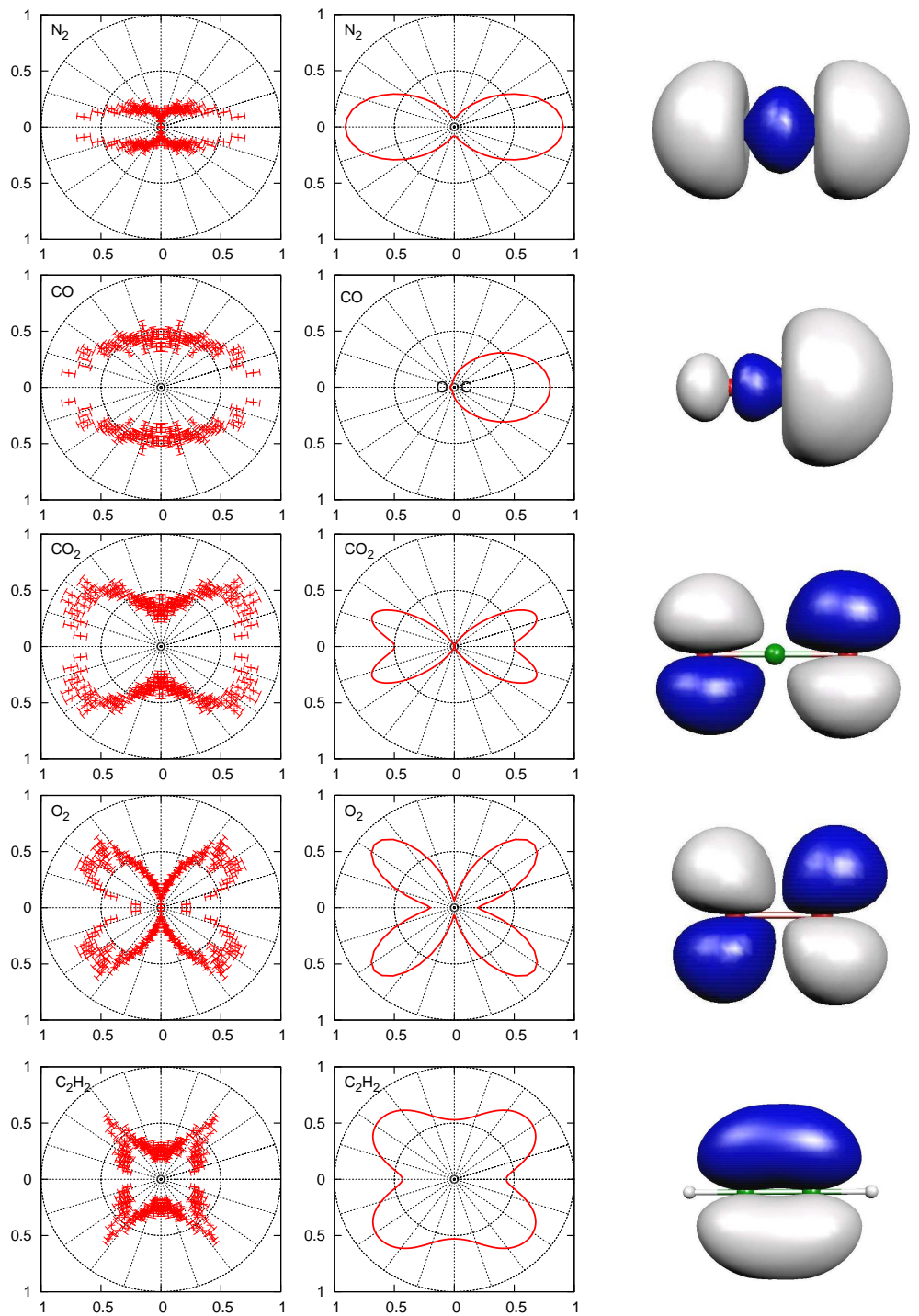

Fig. 1. Comparison of the measured angular distributions of the fragmentation (first column) with the simulated angular distributions of the first ionization (second column) for $\mathrm{N}_{2}, \mathrm{CO}, \mathrm{CO}_{2}, \mathrm{O}_{2}$ and $\mathrm{C}_{2} \mathrm{H}_{2}$ respectively. The corresponding molecular orbitals are also plotted (third column). The experimental data are taken from Refs. [4-6].

In order to extract the alignment-dependent ionization rates of molecules from such experiments, the laser parameters should be in the so-called nonsequential double ionization regime where the second ionization is due to impact ionization by the rescattering electrons. Such electron collisions are known to be fairly isotropic and thus one can approximate the alignment dependence of double ionization to be the alignment dependence of the first ionization alone. Experimentally such conditions are met using sub-10 fs laser pulses at low intensities.

Under these assumptions, we compare in Fig. 1 the angular distributions of 
the measured fragments for a number of molecules taken at Kansas State University [4-7]. Shown are the results for $\mathrm{N}_{2}, \mathrm{O}_{2}, \mathrm{CO}, \mathrm{CO}_{2}$ and $\mathrm{C}_{2} \mathrm{H}_{2}$. For $\mathrm{CO}_{2}$, the breakup channel considered is $\mathrm{CO}_{2}^{2+} \rightarrow \mathrm{CO}^{+}+\mathrm{O}^{+}$and for $\mathrm{C}_{2} \mathrm{H}_{2}^{2+}$ into two $\mathrm{CH}^{+}$ions. These distributions are compared to the alignment-dependent ionization rates of molecules using the MO-ADK theory (2nd column) and with the density distributions of the molecular orbitals (3rd column) from which the electrons have been ionized, calculated from the GAMESS code [8] at the equilibrium distance for each molecule. The calculated electronic wavefunction is visualized by the MOLEKEL program [9].

In Fig. 1 the angular distributions for $\mathrm{N}_{2}$ and $\mathrm{CO}$ are quite similar. The $\mathrm{HOMO}$ of $\mathrm{N}_{2}$ has $\sigma_{g}$ symmetry and of $\mathrm{CO}$ has $\sigma$ symmetry and their angular distributions are peaked in the direction of the laser polarization, in agreement with the fact that the electron density for $\sigma_{g}$ (or $\sigma$ ) orbitals peaks in the direction of the internuclear axis.(The experiments cannot separate $\mathrm{C}-\mathrm{O}$ from $\mathrm{O}-\mathrm{C}$ in the sample so their angular distribution is symmetric.)

In Fig. 1 we also note that the angular distributions for $\mathrm{O}_{2}$ and $\mathrm{CO}_{2}$ are very similar. Their angular distributions peak away from the laser polarization direction. The HOMO of each molecule has $\pi_{g}$ symmetry, and according to the MO-ADK theory the ionization rate peaks when the molecules are aligned at an angle of about $40^{\circ}$ for $\mathrm{O}_{2}$ and $25^{\circ}$ for $\mathrm{CO}_{2}$. The latter has a smaller angle because the $\mathrm{O}-\mathrm{O}$ bond length is larger. The ionization rates show a minimum in the laser polarization direction instead of zero as would be expected for a pure $\pi_{g}$ orbital, reflecting the approximate nature of the MO description of the HOMO orbital.

In Fig. 1 the angular distributions of $\mathrm{C}_{2} \mathrm{H}_{2}$ are shown. For $\mathrm{C}_{2} \mathrm{H}_{2}$, its $\mathrm{HOMO}$ has the $\pi_{u}$ symmetry. Again, the angular distributions measured are in good agreement with the prediction of the MO-ADK theory and with the symmetry property of the $\pi_{u}$ orbital.

The results shown in Fig. 1 clearly indicate that the measurement of alignment dependence of tunneling ionization rates provides an experimental verification of the "physical reality" of molecular orbital (MO). We note that in most quantum measurements the yield is proportional to the square of the matrix element of an operator taken between the initial and final states. Thus it is often difficult to unravel from the measured angular distributions the geometric effect of the initial state and the final state separately. For tunneling ionization of molecules, ionization occurs primarily from electrons initially in the direction of the electric field. In the tunneling process the ionized electrons remain in the direction of the electric field. By detecting the breakup of the molecular ion thus gives a direct measurement of the electron density distribution of the outermost orbital if the molecular axis does not undergo additional rotation in the breakup process. These conditions are met for non-sequential 
double ionization of simple molecules by sub-10 fs laser pulses.

We comment that there do exist discernable disagreement between the MOADK theory and experimental results. The limitation of the theory includes that we ignored the possible small contributions from inner molecular orbitals, which will make the region near the nodal surfaces less pronounced.

\section{Probing and imaging simple molecules with molecular high-order harmonic generation}

High-order harmonic generation (HHG) is understood in terms of the threestep model: At the peak of the electric field of the laser, electrons are ionized by tunneling. These electrons are then accelerated in the laser field and are driven back to recollide with the ions when the laser's electric field reverses its direction. HHG is emitted when the returned electrons recombine with the ions. The second step is known to be fairly isotropic because of the returning wave packet is sufficiently broadened, but tunneling ionization and recombination both are expected to depend on the structure of molecules. Thus alignment dependence is expected for the HHG emission.

In the last few years there have been quite a few experimental investigations[1014] of the alignment dependence of HHG using the pump-probe arrangement: A weak pump beam was used first to create field-free time-dependent dynamic alignment of molecules. A second intense short pulse is used to generate HHG during the short time interval when the molecules are maximally aligned. The angle between the polarization vectors of the two beams can be varied, or the two polarizations are kept parallel but HHG were determined for the different time delays.

So far HHG from such experiments have been reported for $\mathrm{N}_{2}, \mathrm{O}_{2}$ and $\mathrm{CO}_{2}$ molecules. For $\mathrm{N}_{2}$ molecules, the dependence of HHG on the angle between the pump and probe laser beams had been determined and the angular dependence of HHG was used to extract the wavefunction of the outermost occupied molecular orbital of $\mathrm{N}_{2}$, using the tomographic imaging method[10]. For $\mathrm{O}_{2}$ and $\mathrm{CO}_{2}$, they behave differently. For $\mathrm{CO}_{2}$, in particular, it has been shown that the harmonic yield at certain order shows inversion or minimum[13,14]. The minimum has been interpreted in terms of the two-center interference model[15], i.e., the returning electron wave packet arriving at the two atomic centers has a phase difference and this phase difference depends on the alignment angle of the molecule with respect to the laser polarization direction (pump and probe beams are collinear). On the other hand, the details of how the two-center interference model works is still controversial. 
In the typical pump-probe experiments carried out so far, the degree of alignment of the molecules from the pump pulse is limited. There is still a fairly wide angular distributions of molecules even during the short intervals when the molecules are maximally aligned. Thus the harmonic yields should be averaged over these angular distributions. Such averages have not been accounted for in the experimental analysis of the observed data so far.

Theoretical calculations of the alignment-dependent HHG yields for such complex molecules are not available. For the simplest $\mathrm{H}_{2}^{+}$, ab initio calculations for the alignment dependence have been carried out recently[16], but the computational effort is still too large to explore the typical pump-probe experiments. A simpler model calculation is needed. In Zhou et al $[17,18]$, $\mathrm{HHG}$ from $\mathrm{N}_{2}$ and $\mathrm{O}_{2}$ molecules have been calculated using the Lewenstein model. The model of Lewenstein et al. [19] for the high order harmonic generation from atoms was based on the strong field approximation. This model was obtained by expanding the time-dependent wavefunction in terms of the basis functions of the atom, with the laser-atom interaction providing the transitions. By truncating the Hilbert space to including only the ground state and excluding all the other bound excited states, and further approximating the continuum states by the Volkov states, the time-dependent dipole moment for an atom in the laser field $E(t)$ was expressed as [19]

$$
\begin{aligned}
r_{\mathbf{n}}(t)= & i \int d t^{\prime} \int d^{3} \mathbf{p} \mathbf{n} \cdot \mathbf{d}^{*}(\mathbf{p}-\mathbf{A}(t)) a^{*}(t) \\
& \mathbf{E}\left(t^{\prime}\right) \cdot \mathbf{d}\left(\mathbf{p}-\mathbf{A}\left(t^{\prime}\right)\right) a\left(t^{\prime}\right) e^{-i S\left(\mathbf{p}, t, t^{\prime}\right)}+\text { c.c. }
\end{aligned}
$$

where the laser polarization direction is along $\mathbf{n}$ and c.c. stands for complex conjugate. In Eq. (1), $a(t)$ is the amplitude of the ground state, $\mathbf{d}(\mathbf{p}-\mathbf{A}(t))$ is the transition dipole moment between the ground state and the continuum state, with $\mathbf{p}$ and $\mathbf{A}$ the canonical momentum and vector potential, respectively, and

$$
S\left(\mathbf{p}, t, t^{\prime}\right)=\int_{t^{\prime}}^{t} d t^{\prime \prime}\left[\frac{1}{2}\left(\mathbf{p}-\mathbf{A}\left(t^{\prime \prime}\right)\right)^{2}+I_{p}\right]
$$

is the quasiclassical action, where $I_{p}$ is the ionization potential of the atom. By comparing the prediction from the Lewenstein model for atoms with those from actual numerical calculations, it has been shown that the model can predict HHG yield in the plateau region well, but not for the lower-order HHG's.

To calculate the HHG from aligned molecules, the ground state molecular wavefunction is obtained from the GAMESS code, where each molecular orbital is expanded in terms of atomic orbitals on each center. 

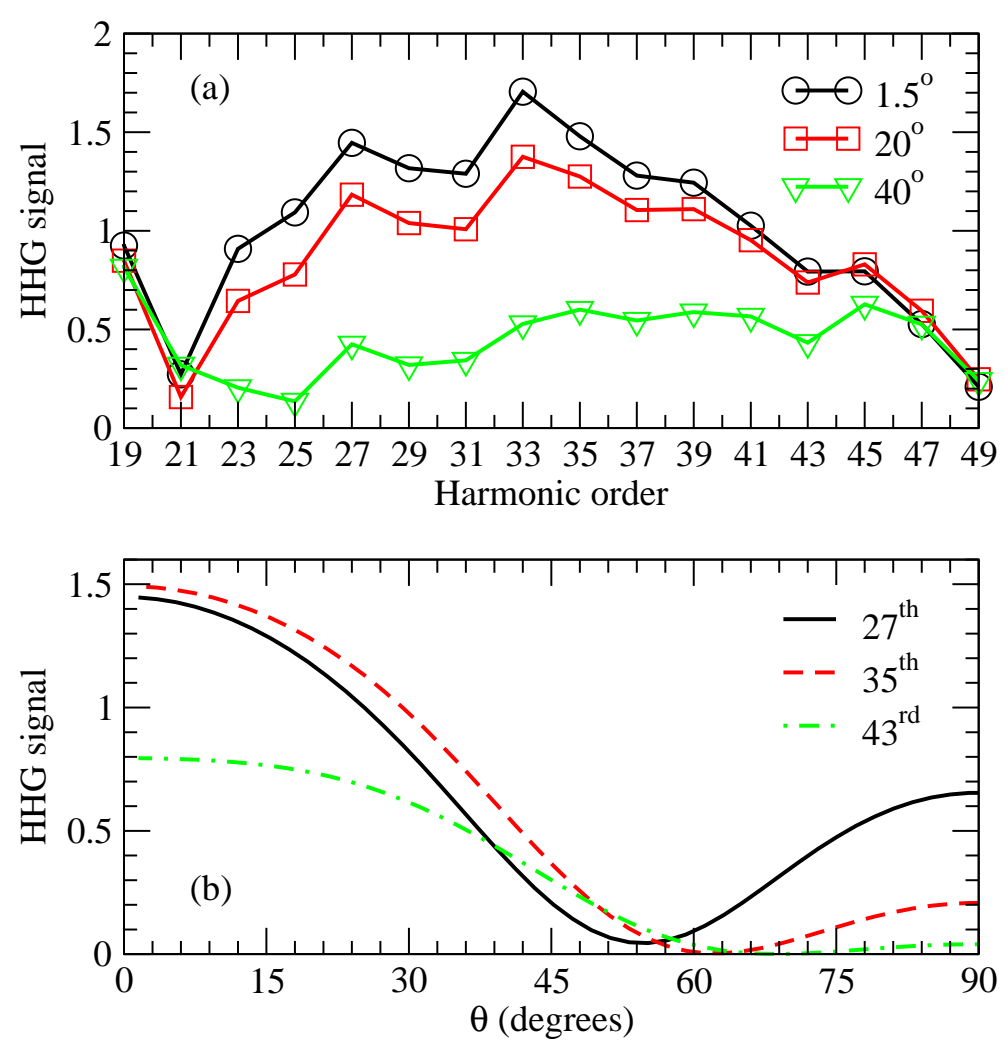

Fig. 2. (Color online) Alignment dependence of the HHG signals from $\mathrm{N}_{2}$ calculated using the Lewenstein model. Laser pulse duration, $30 \mathrm{fs}$, peak intensity, $3 \times 10^{14}$ $\mathrm{W} / \mathrm{cm}^{2}$, and mean wavelength, $800 \mathrm{~nm}$.

Using the Lewenstein model, the alignment dependence of HHG from $\mathrm{N}_{2}$ and $\mathrm{O}_{2}$ have been calculated. Fig. 2 and Fig. 3 show the HHG yields for different orders at a given alignment angle of the molecule, and the angular dependence of a specific harmonic, respectively. Clearly the alignment dependence of the yield for each specific harmonic is different for $\mathrm{N}_{2}$ as compared to $\mathrm{O}_{2}$.

One can compare the alignment dependence of the HHG yield vs the alignment dependence of the tunneling ionization rates as depicted in Fig. 1. Since the first step of the harmonic generation is tunneling ionization, such comparison would give some information about the alignment dependence of the recombination process. For $\mathrm{N}_{2}$, it is clearly seen that both the HHG yield and the tunneling ionization rate peak at $\theta=0$. However, there is some difference at larger angles where the ionization rate is small but the HHG yield actually increases. There are two reasons for this difference. First, tunneling ionization, as indicated in the previous section, depends on the electronic wavefunction in the outer region. For the recombination process, the wavefunction in the inner region is sampled and the hybridization of the molecular orbital is more important. Thus the $\sigma_{g}$ orbital has contributions from $2 \mathrm{p} \sigma$ as well as from $2 \mathrm{~s} \sigma$ orbitals. For the tunneling ionization, it is the wavefunction at large distance that is sampled where hybridization from the $2 \mathrm{~s} \sigma$ orbital is negligible. There 

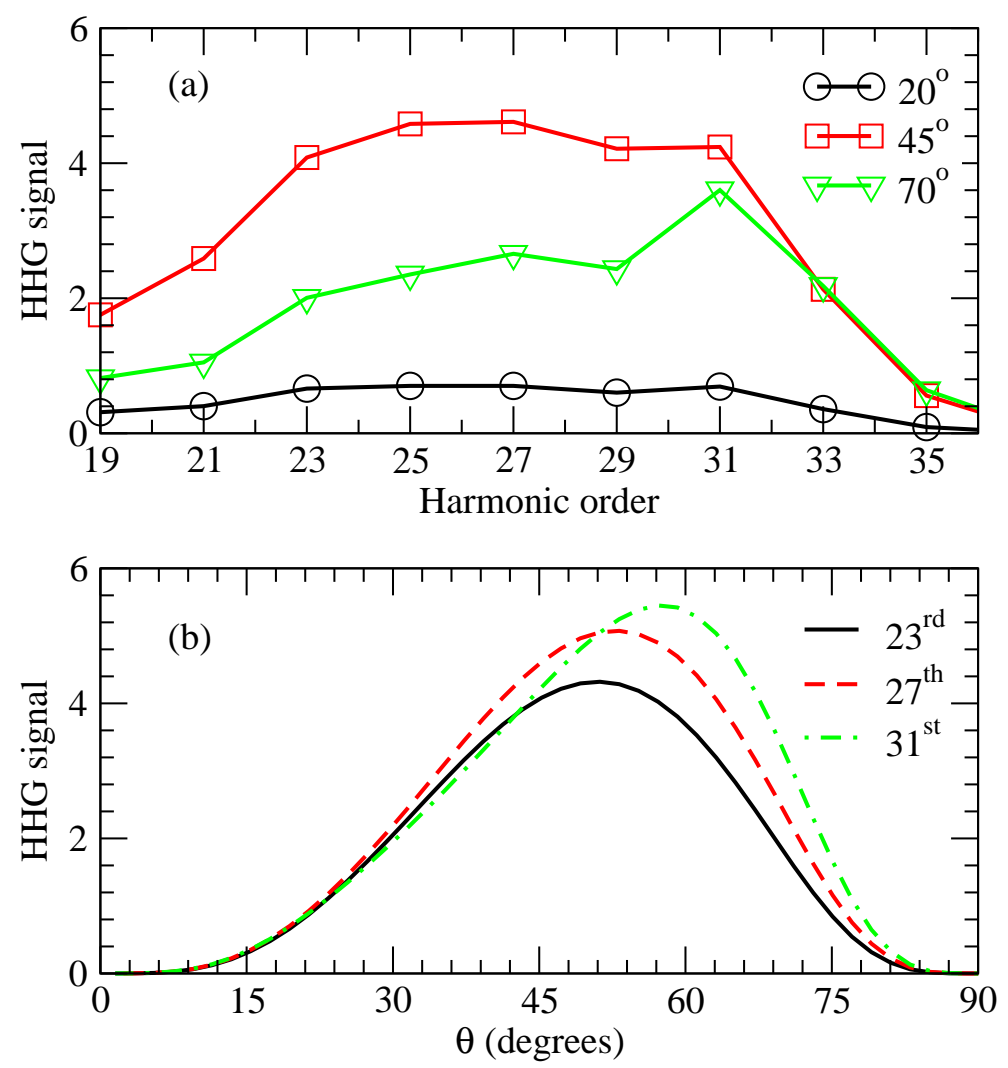

Fig. 3. (Color online) Alignment dependence of the HHG signals from $\mathrm{O}_{2}$ calculated using the Lewenstein model. Laser pulse duration, $30 \mathrm{fs}$, peak intensity, $2 \times 10^{14}$ $\mathrm{W} / \mathrm{cm}^{2}$, and mean wavelength, $800 \mathrm{~nm}$.

is another factor that can contribute to the larger HHG yield at larger alignment angles as shown in Fig. 2(b)- the depletion of the ground state. Recall that the ionization rate is smaller at larger angles. For the intensity of $3 \times 10^{14}$ $\mathrm{W} / \mathrm{cm}^{2}$, it was found that depletion is significant. The HHG yield at larger angles has been found to be affected by the depletion. In Fig. 3 for $\mathrm{O}_{2}$, the angles where the HHG yield peaks are much larger than the angle where the tunneling ionization rate peaks, at about $42^{\circ}$. The shift to the larger angles actually have been shown mostly due to the depletion effect at the laser intensity of $2 \times 10^{14} \mathrm{~W} / \mathrm{cm}^{2}$. Without the depletion, the peak in HHG is closer to about $50^{\circ}$.

The HHG yield at a fixed angle between the molecular axis and the laser polarization direction cannot be obtained experimentally so far. In the pumpprobe experiment, a weak pump pulse was used to align the molecules, and then a probe pulse was used to generate HHG at different time delays. The degree of alignment of the molecules after the pump pulse changes with the time delay. At the time of full or fractional rotational periods, the molecules undergo maximum alignment. The degree of alignment is measured in general by the expectation value $\left\langle\cos ^{2} \theta>\right.$. In Fig. 4 we show the comparison of the calculated HHG yield vs the delay time for the 23rd, 27th and 31st harmonics 


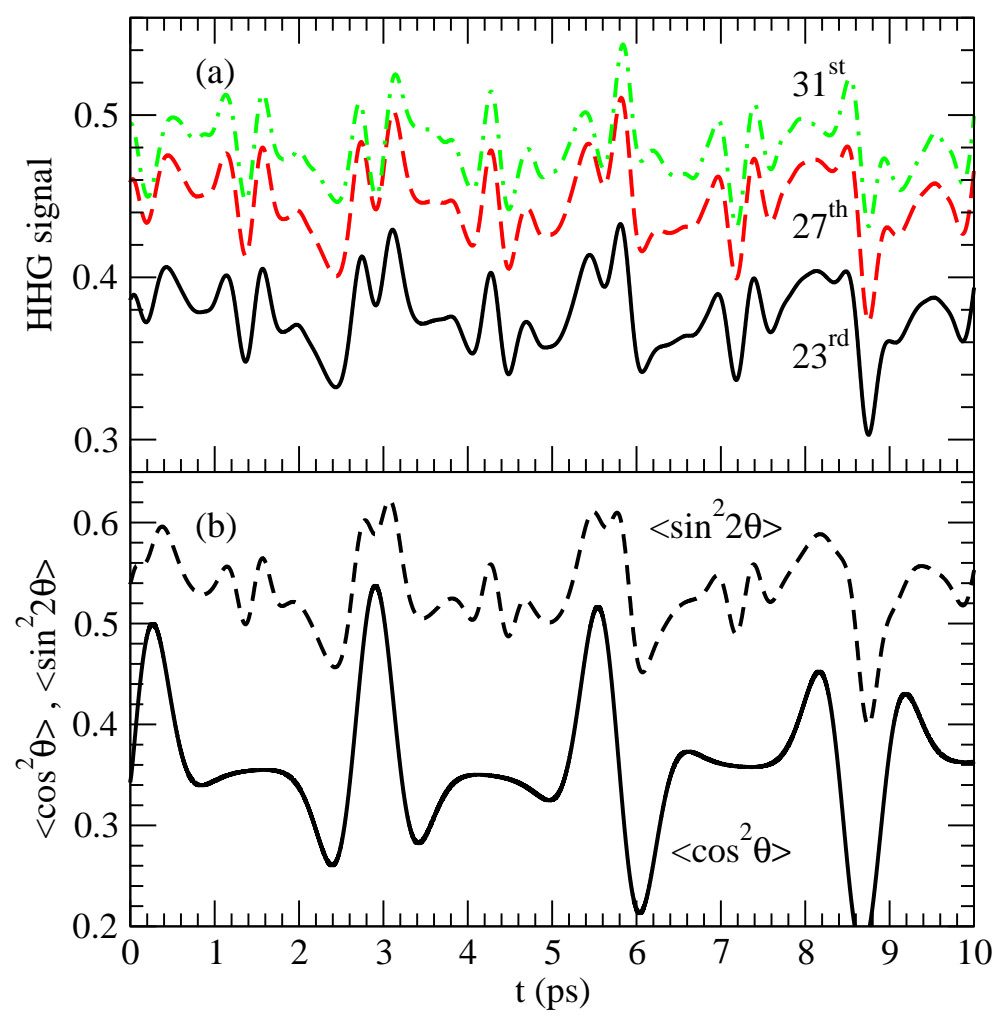

Fig. 4. (Color online) High-order harmonic yields generated by a probe pulse after the ensemble of molecules have been dynamically aligned by a pump laser pulse. The yield of each harmonic (a) vs time is shown not to follow the average alignment $\left\langle\cos ^{2} \theta\right\rangle$ of the molecules, but shifted by about $45^{\circ}$, as measured by $\left\langle\sin ^{2} 2 \theta\right\rangle$, (b). HHG signals: 23rd(black), 27th(red), 31st(Green), for $\mathrm{O}_{2}$ molecules.

of $\mathrm{O}_{2}$. It is clear that the time dependence of the HHG yields is different from the expectation value $\left\langle\cos ^{2} \theta>\right.$. In fact, they are closer to the expectation values of $<\sin ^{2}(2 \theta)>$. This shows that the HHG yield tends to peak near $45^{\circ}$. At this level of comparison, the predicted detailed angular distribution cannot be extracted from the experimental data. On the other hand, the alignment dependence of different molecules clearly show the dependence on the geometric property of the molecular orbital.

\section{Probing vibrational wave packets using attosecond pulses}

Picosecond and femtosecond lasers have been used to determine the motion of the vibrational wave packets of large molecules using various pump-probe techniques. Obviously, to determine the wave packet motion of the simplest molecules like $\mathrm{H}_{2}^{+}$and $\mathrm{D}_{2}^{+}$, where the vibrational periods are about 15 fs and $22 \mathrm{fs}$, respectively, sub-10fs pulses would be needed. In a recent experiment, Alnaser et al. [20] used two successive $\amalg 8$ spulsestodoubleionize $\mathrm{H}_{2}$ and $\mathrm{D}_{2}$. By measuring the kinetic energy release of the two atomic ions after the second 


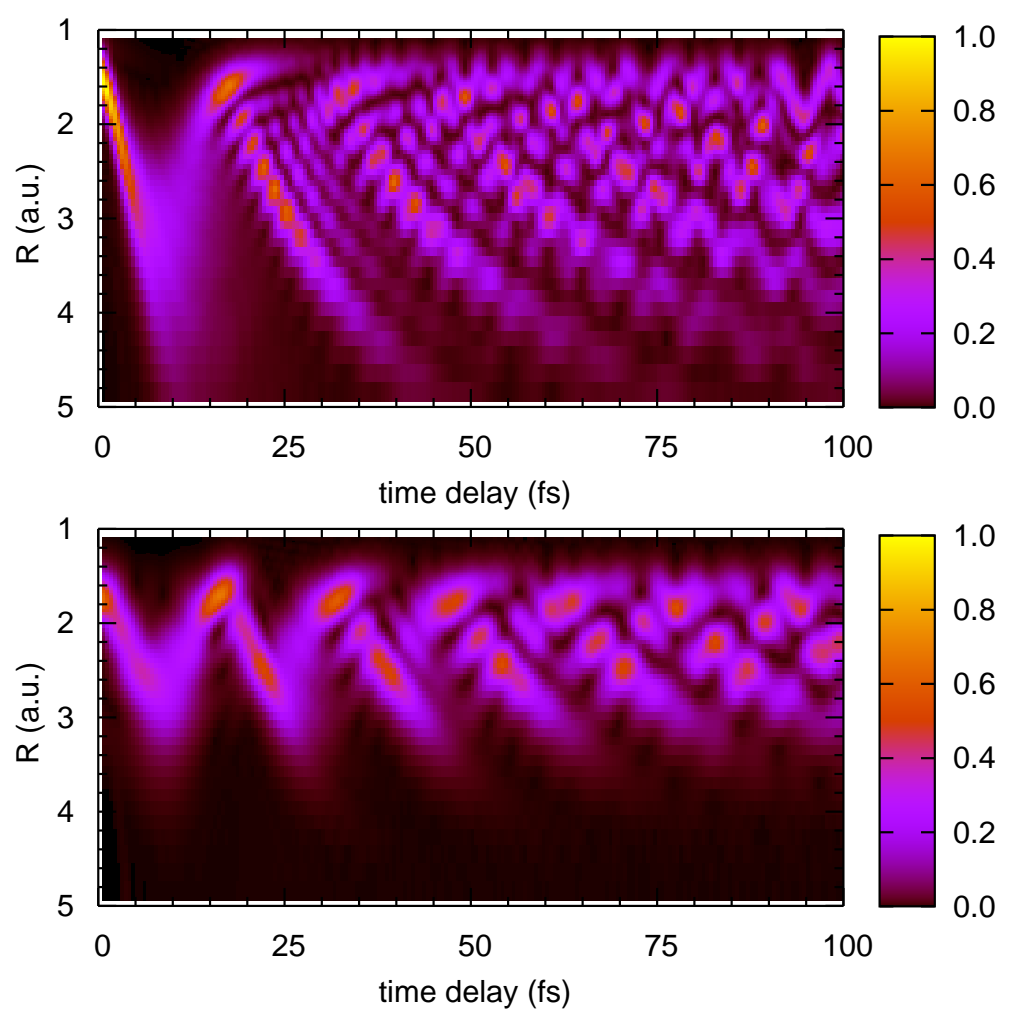

Fig. 5. Time evolution of the density distribution of the $\mathrm{H}_{2}^{+}$vibrational wave packet after ionizing $\mathrm{H}_{2}$ at $\tau=0$. (a) assuming Franck-Condon distribution (top frame) and (b) by using the vibrational distribution from experiment [21] (bottom frame).

ionization from the probe pulse, "movies"showing the motion of the wave packets on the lowest two potential curves of the molecular ions have been reconstructed.

Theoretically the time-dependent development of the wave packet can be trivially calculated for the present systems if the initial wave packet is known. Fig. 5 show an example of the time-evolution of the two wave packets of $\mathrm{H}_{2}^{+}$. One of the wave packet was created by ionizing $\mathrm{H}_{2}$ at $\mathrm{t}=0$ assuming that the initial vibrational distribution follows the Franck-Condon (F-C) principle. For the other wave packet the initial vibrational distribution was assumed to be given by the weight measured by Urbain et al[21], but with the same phases as given by the $\mathrm{F}-\mathrm{C}$ principle. One can see that the time evolution of the two vibrational wave packets are clearly distinguishable.

In Fig. 6 we show the reconstructed wave packet evolution if the wave packets are probed with an 8 fs laser pulse, or with an attosecond pulse of duration of $0.5 \mathrm{fs}$, with mean energy of $30 \mathrm{eV}$, assuming that the initial wave packet follows the F-C principle. For the 8 fs probe, the predicted stripes toward large $\mathrm{R}$, for the range of $\mathrm{R}$ greater than about $2.2 \mathrm{a} . \mathrm{u}$, are clearly observed, but the details, as well as the interference patterns, are hardly visible. Note that the wave packet behaves drastically different from what is expected from classical 


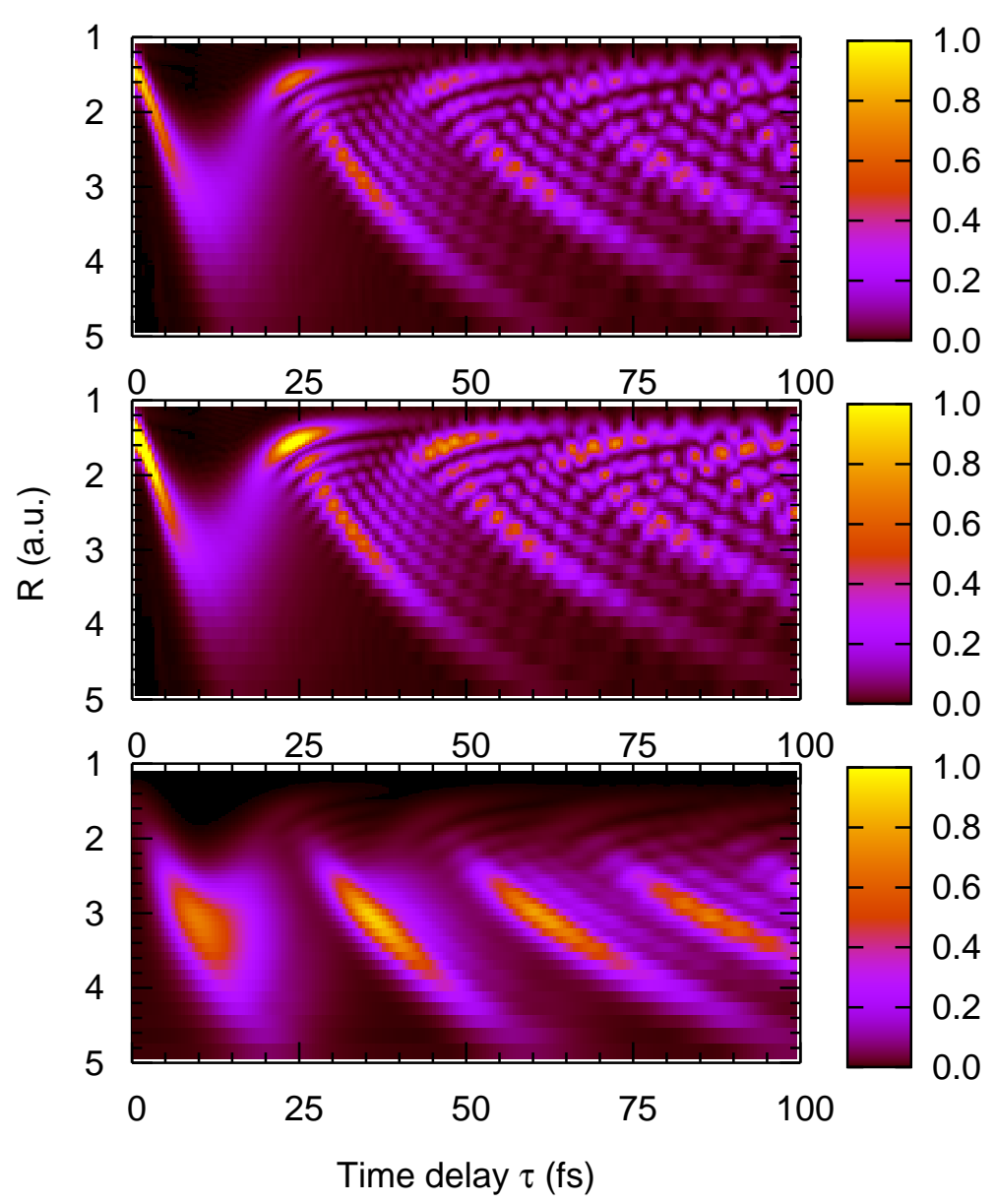

Fig. 6. Time evolution of the density distribution of the $\mathrm{D}_{2}^{+}$vibrational wave packet after ionizing $\mathrm{D}_{2}$ at $\tau=0$, assuming Franck-Condon principle. (a) "Exact" theoretical wave packet (top frame); (b) Reconstructed wave packet from probing with a 0.3 fs XUV pulse with center energy at $50 \mathrm{eV}$ (middle frame); and (c) Reconstructed wave packet from probing with an $8 \mathrm{fs}, 800 \mathrm{~nm}$ IR laser with intensity $9 \times 10^{14} \mathrm{~W} / \mathrm{cm}^{2}$ (bottom frame). The middle frame shows that wave packets are reconstructed in verbatim when probed with attosecond XUV pulses.

physics. The wave packet is not localized during the second half of each vibrational period, as predicted from the theoretical simulation, and this can be seen from the simulated probe signal by the $8 \mathrm{fs}$ pulse, see the bottom frame of Fig. 6. This theoretical simulation agrees very well with the experimental results of Alnaser et al. In Fig. 6 we note that the wave packet extracted using the attosecond probe reproduces all the details of the theoretically calculated wave packet, including the features of the interference. With such details, it is possible to deduce the initial vibrational level populations, including the phases of each vibrational state, following the pump pulse. This indicates that attosecond pulses could be used as powerful tool for probing the details of an experiment. In turn, such measurements would allow the determination on how the vibrational wave packet depends on the laser parameters of the pump pulse directly, for example, by using shaped pulses. 


\section{Summary and Discussion}

In this paper we gave three examples of how the femtosecond laser or attosecond XUV pulses can be used to probe the structure of simple molecules. Using sub-10fs laser pulses with proper intensity, the nature of the highest occupied molecular orbital (HOMO) can be directly probed and visualized from the angular distribution of the breakup of molecular ions into two ion fragments. Similarly, the high-order harmonic generation (HHG) is shown also to depend on the nature of the molecular structure, in particular, the geometry of the HOMO, as well as the internuclear separations. Thus tunneling ionization and the HHG can be used to probe the structure of simple molecules. In particular, with such short pulses, the time-evolution of these simple molecules in their breakup or in their reaction with other molecules or with the environment can be probed with similar means in the future. We also illustrate that attosecond light pulses can be used to study the details of the vibrational wave packet for simple molecules. With such tools, the pulse acts like a "white" light, and the wave packet can be probed with fine details. Such time-dependence information may be "inverted " to extract the phase and amplitude of each inelastic reaction product after the pump pulse. With such detailed information, it is further possible to modify the pump pulse to generate specific final output desired. The future of ultrashort laser and/or light pulses is bright and we can look forward to exciting further applications in the years to come.

CDL was supported in part by Chemical Sciences, Geosciences and Biosciences Division, Office of Basic Energy Sciences, Office of Science, U. S. Department of Energy.

\section{References}

[1] H. Stapelfeldt, T. Seideman, Rev. Mod. Phys. 75 (2003) 543.

[2] X. M. Tong, Z. X. Zhao, C. D. Lin, Phys. Rev. A 66 (2002) 033402.

[3] M. V. Ammosov, N. B. Delone, V. P. Krainov, Zh. Eksp. Teor. Fiz. 91 (1986) 2008-2013, [Sov. Phys. JETP 64 (1986) 1191].

[4] A. S. Alnaser, S. Voss, X. M. Tong, C. M. Maharjan, P. Ranitovic, B. Ulrich, T. Osipov, B. Shan, Z. Chang, C. L. Cocke, Phys. Rev. Lett. 93 (2004) 113003.

[5] S. Voss, A. S. Alnaser, X. M. Tong, C. Maharjan, P. Ranitovic, B. Ulrich, B. Shan, Z. Chang, C. D. Lin, C. L. Cocke, J. Phys. B 37 (2004) 4239-4258.

[6] A. S. Alnaser, C. M. Maharjan, X. M. Tong, B. Ulrich, P. Ranitovic, B. Shan, Z. Chang, C. D. Lin, C. L. Cocke, I. V. Litvinyuk, Phys. Rev. A 71 (2005) 031403 . 
[7] X. M. Tong, Z. X. Zhao, A. S. Alnaser, S. Voss, C. L. Cocke, C. D. Lin, J. Phys. B 38 (2005) 333-341.

[8] M. W. Schmidt, K. K. Baldridge, J. A. Boatz, S. T. Elbert, M. S. Gordon, J. J. Jensen, S. Koseki, N. Matsunaga, K. A. Nguyen, S. Su, T. L. Windus, M. Dupuis, J. A. Montgomery, J. Comput. Chem. 14 (1993) 1347-1363.

[9] S. Portmann, H. P. Lüthi, CHIMIA 54 (2000) 766-770.

[10] J. Itatani, J. Levesque, D. Zeidler, H. Niikura, H. Pepin, J. C. Kieffer, P. B. Corkum, D. M. Villeneuve, Nature 432 (2004) 867-871.

[11] J. Itatani, D. Zeidler, J. Levesque, M. Spanner, D. M. Villeneuve, P. B. Corkum, Phys. Rev. Lett. 94 (2005) 123902.

[12] M. Kaku, K. Masuda, K. Miyazaki, Jpn. J. Appl. Phys. 43 (2004) L591-L593.

[13] T. Kanai, S. Minemoto, H. Sakai,, Nature 435 (2005) 470-474.

[14] C. Vozzi, F. Calegari, E. Benedetti, J.-P. Caumes, G. Sansone, S. Stagira, M. Nisoli, R. Torres, E. Heesel, N. Kajumba, J. P. Marangos, C. Altucci, R. Velotta, Phys. Rev. lett. 95 (2005) 153902.

[15] M. Lein, N. Hay, R. Velotta, J. P. Marangos, P. L. Knight, Phys. Rev. A 66 (2002) 023805.

[16] G. Lagmago Kamta and A. D. Bandrauk, Phys. Rev. A 71 (2005) 053407.

[17] X. X. Zhou, X. M. Tong, Z. X. Zhao and C. D. Lin, Phys. Rev. A 71 (2005) 061801(R).

[18] X. X. Zhou, X. M. Tong, Z. X. Zhao, C. D. Lin, Phys. Rev. A 71 (2005) 061801.

[19] M. Lewenstein, P. Balcou, M. Y. Ivanov, A. Lhuillier, P. B. Corkum, Phys. Rev. A 49 (1994) 2117-2132.

[20] A. S. Alnaser et al., Phys. Rev. A 72 (2005) 030702(R).

[21] X. Urbain, B. Fabre, E. M. Staicu-Casagrande, N. de Ruette, V. M. Andrianarijaona, J. Jureta, J. H. Posthumus, A. Saenz, E. Baldit, C. Cornaggia, Phys. Rev. Lett. 92 (2004) 163004. 